\title{
Evolución del sistema de salud de Ecuador. Buenas prácticas y desafíos en su construcción en la última década 2005-2014
}

\section{Evolution of the health system of Equator, good practices and challenges in its construction in the last decade 2005-2014}

\author{
Caroline J. Chang Campos ${ }^{1}$ \\ ${ }^{1}$ Phd en Ciencias de la Salud, MSc en Gerencia en salud para el desarrollo local, Especialista en gerencia de servicios de salud, Training in international health, Doctora en \\ Medicina y Cirugía, EsSalud, Lima, Perú.
}

Correspondencia

Caroline Chang Campos

carolinejchangc@gmail.com

Recibido: 25 de julio 2016.

Aceptado: 11 de enero 2017.

Conflictos de interés: La autora se desempeñó como Ministra de Salud Pública del Ecuador en el período de enero 2007 a abril 2010.

Financiamiento: Financiamiento propio de la autora.

Citar como: Chang Campos CJ. Evolución del sistema de salud de Ecuador. Buenas prácticas y desafios en su construcción en la última década 2005-2014. An Fac med. 2017;78(4):452-60 DOI: $h$ ttp://dx.doi.org/10.15381/ anales.v78i4.14270
An Fac med. 2017;78(4):452-60 / http://dx.doi.org/10.15381/anales.v78i4.14270

Resumen

Introducción. Ecuador ha emprendido importantes reformas en búsqueda de la universalidad y equidad, sin embargo, el sistema continúa fragmentado y no garantiza el acceso universal. Este estudio pretende abordar desde la perspectiva de las representaciones sociales, la evolución del sistema de salud del Ecuador a fin de contribuir a los procesos de reforma del país y de la Región. Objetivo. Describir la rectoría, financiamiento y prestación de servicios relacionados con los factores políticos, sociales y económicos, identificar las buenas prácticas, lecciones aprendidas y desafíos en la construcción. del sistema. Metodología. Estudio cualitativo descriptivo-interpretativo basado en la Teoría de las Representaciones Sociales, mediante revisión sistemática de documentos y entrevistas a profundidad a 14 actores involucrados en los procesos de reformas, las mismas que fueron codificadas, analizadas e interpretadas, destacando las percepciones mayoritarias, convergencias y divergencias. Resultados y Conclusiones. El sistema de salud es por mandato constitucional de acceso universal y gratuito. Las representaciones sociales reconocen la rectoría de la autoridad sanitaria que requiere fortalecer el liderazgo y legitimización por parte del sector y sociedad civil. Posicionar a la salud como derecho y prioridad de Estado ha sido una de las mejores buenas prácticas, mientras que la falta de participación social para la legitimización y validación de los procesos de cambios es considerada una lección aprendida, ya que si bien es cierto los factores políticos, sociales y económicos a partir del 2007 han sido favorables para la transformación del sector salud, la alteración o revés de estos factores pondría en riesgo los avances y consolidación del Sistema de Salud. El principal desafío es la sostenibilidad financiera de la universalidad y gratuidad en consonancia con la calidad, siendo necesaria la racionalización del gasto y orientar la prestación de servicios al modelo de atención basado en atención primaria de salud.

Palabras clave: Sistemas de salud; Investigación cualitativa.

\section{Abstract}

Introduction. Ecuador has undertaken important reforms in search of universality and equity however; the system continues fragmented and does not guarantee universal access. This study aims to address the evolution of the health system of the Ecuador in order to contribute with the process the country and the Region reform from the perspective of social representations Objective. Describe the governance, financing and provision of services related to political, social and economic factors, identify good practices, lessons learned and challenges in construction of the system. Methodology. Qualitative descriptive-interpretative study based on the Theory of Social Representations, through systematic review of documents and in-depth interviews with 14 actors involved in the reform process, which were codified, analyzed and interpreted, highlighting the majority perceptions, convergences and divergences. Results and Conclusions. The health system is universal and of free access by constitutional mandate. The social representations recognize the governance of the health authority that requires strengthening the leadership and legitimization on the part of the sector and of the civil society. Positioning Health as "right" and priority of the State, it has been one of the best "good practices", while the lack of social participation for the legitimization and validation of change processes is considered a lesson learned, since it is true the political, social and economic factors have been favorable for the transformation of the health sector since 2007. The alteration or setback of these factors would jeopardize the progress and consolidation of the Health System. The main challenge is the financial sustainability of universality and free of charge in line with quality, being necessary the rationalization of expenditure and the guided services to the care model based on primary health care.

Keywords: Health systems; Qualitative research. 


\section{INTRODUCCIÓN}

Los sistemas de salud son el conjunto de relaciones políticas, económicas e institucionales responsables por la conducción de los procesos relativos a la salud de la población, que se expresan en organizaciones, normas y servicios, que tienen como objetivo alcanzar resultados consistentes con la concepción de salud prevalente en la sociedad ${ }^{(1)}$. Según la OMS, el funcionamiento de un sistema de salud contempla principalmente: La rectoría, provisión de los servicios de salud, financiamiento y recursos humanos (2). Cada país construye su sistema de salud, basado en los modelos de desarrollo y determinantes políticos, sociales y culturales.

En las últimas décadas los países de América Latina han experimentado importantes reformas en los sistemas de salud, y a pesar de los esfuerzos y avances positivos en procesos de descentralización y participación en salud, en su mayoría no han logrado los objetivos de disminución de inequidades, mayor eficiencia, y mejora de la calidad de los servicios. En el Ecuador en la última década los procesos de reformas y transformación del sector salud (TSSE) han sido la agenda prioritaria de los gobiernos y ministros de salud.

Desde el 2005 al 2007 se impulsó el Aseguramiento Universal en Salud (AUS) que seguía los lineamientos internacionales propuestos por el Banco Mundial. A partir del 2007 se inició el proceso de transformación del sector salud hacia el acceso universal gratuito, y es en el 2008 que la nueva Constitución de la República ${ }^{(3,4)}$ declara a la salud como un derecho humano inalienable y al Estado como garante del acceso universal y gratuito de la población para la atención y prevención de enfermedades. A pesar de los importantes esfuerzos y avances en la universalidad, el sistema no garantiza totalmente el acceso universal, y encontramos fragmentación, exclusión en las prestaciones, dificultad en el financiamiento y en el acceso a los servicios de salud.

El estudio cualitativo pretende describir las principales características y la evolución del sistema de salud de Ecuador en materia de rectoría, prestación de servicios y financiamiento; además de relacionar desde la perspectiva de los informantes si las reformas o cambios en el sistema de salud han logrado mejorar el acceso a los servicios y si están o no contribuyendo a reducir la inequidad. Es objetivo también del estudio, identificar las buenas prácticas, lecciones aprendidas y desafíos en la construcción del sistema de salud de Ecuador en la década 2005 - 2014, comprenderlos e interpretarlos en base a las representaciones sociales a la luz de los factores políticos, sociales y económicos, considerando que las decisiones políticas de alto nivel, los determinantes económicos y la participación ciudadana son elementos claves para estos avances y al mismo tiempo limitaciones para la sostenibilidad de los sistemas y su permanente perfeccionamiento.

\section{METOdOLOGía}

El abordaje metodológico del estudio sobre la evolución del sistema de salud del Ecuador fue cualitativo, descriptivo-interpretativo, utilizando la Teoría de las Representaciones Sociales, para identificar las características, buenas prácticas, lecciones aprendidas y desafíos de los procesos y reformas del sistema de salud del Ecuador, así como los factores políticos, sociales y económicos que influyeron en dichos procesos.

El estudio cuenta con una revisión documentaria basada en datos y estadísticas publicadas en fuentes oficiales o de organismos internacionales y el análisis con enfoque cualitativo de las percepciones y reflexiones convergentes y divergentes, obtenidas en las entrevistas a profundidad a las autoridades, expertos y actores involucrados en los procesos de reforma durante el período de estudio.

Para la revisión sistemática se utilizó una guía de descripción de sistemas de salud que fue adaptada de la guía diseñada por ISAGS/UNASUR para orientar al análisis crítico de los sistemas de salud en Sudamérica.

Para la descripción y análisis de las variables se utilizó la tipología para descripción de los sistemas de salud propuesta por la OMS, en la que se incluye la rec- toría, prestación de servicios, y financiamiento de los sistemas de salud.

Las entrevistas permitieron recoger las opiniones, reflexiones, valoraciones y tendencias de los procesos desarrollados en la última década en la construcción del sistema de salud del Ecuador, se seleccionaron 14 informantes de los cuales 8 (57\%) fueron autoridades durante el periodo de estudio incluyendo algunos en ejercicio del cargo, es así que se entrevistó a 2 ministros de salud pública, 1 Secretario de Estado, 2 viceministros de salud, 1 subsecretario, 1 subsecretario y asambleísta, 1 Director de Salud de la Seguridad Social y Director de Salud de las Fuerzas Armadas; $2(14,3 \%)$ representantes de organismos internacionales, 2 $(14,3 \%)$ académicos, $2(14,3 \%)$ representantes de la sociedad civil de los cuales 1 de ellos fue Director del Consejo Nacional de Salud (CONASA).

Para las entrevistas se diseñó y aplicó una encuesta, matriz de codificación y categorización ${ }^{(5,6)}$, las opiniones y comentarios que se registraron en este estudio corresponden a cada entrevistado que ha sido codificado con la letra $\mathrm{E}$ y numerado de acuerdo a una lista elaborada. Mediante consentimiento informado, los entrevistados autorizaron la publicación de los resultados siempre respetando el anonimato de los testimonios.

La teoría de las representaciones sociales es un proceso de construcción de la realidad a través de la cual quien conoce se coloca dentro de lo que conoce, donde fenómenos de la cultura, la ideología, y la pertenencia socio-cultural dejan su impronta y los elementos afectivos, cognitivos, simbólicos y valorativos participan en su configuración ${ }^{(7,8)}$.

\section{RESULTADOS Y DISCUSIÓN}

\section{Características del Sistema de Salud}

El sistema nacional de salud del Ecuador (SNS) es un sistema mixto, segmentado y fragmentado. El sector público está conformado por el Ministerio de Salud Pública (MSP) que es el principal prestador de servicios, por el Instituto Ecuatoriano de Seguridad Social (IESS) que cotiza a los tra- 
bajadores formales y por los Institutos de Seguridad de las Fuerzas Armadas (ISFA) y Policía Nacional (ISPOL), además de las redes de salud de gobiernos autónomos descentralizados como municipios y prefecturas, la Junta de Beneficencia de Guayaquil (JB), la Sociedad Protectora de la Infancia de Guayaquil, la Sociedad de Lucha contra el Cáncer (SOLCA) y la Cruz Roja Ecuatoriana. El sector privado lo conforman las entidades con fines de lucro (hospitales, clínicas, consultorios), ONG's y asociaciones de servicio social y medicina prepagada, el sector privado representa el $15 \%$ de los establecimientos de salud del país ${ }^{(9)}$.

\section{Reformas del Sistema de Salud}

En el 2005 se impulsó desde la presidencia de Alfredo Palacios el programa de Aseguramiento Universal en Salud (PROAUS) que promovió una política nacional en aseguramiento en salud a través de mecanismos de compra de servicios. El objetivo de PRO-AUS fue brindar un sistema de aseguramiento que comprendía prestaciones integrales con calidad, eficiencia y equidad, bajo una concepción de protección social y una lógica de aseguramiento público, priorizando a la población que vive en condiciones de extrema pobreza y pobreza ${ }^{(9)}$. El AUS impulsó la descentralización de los servicios de salud a los municipios desarrollando en Guayaquil, Quito y Cuenca programas de aseguramiento en salud.

En la primera fase del AUS se preveía alcanzar para 2010 la cobertura universal para las poblaciones de los quintiles 1 y 2 de menores ingresos a través del Programa de extensión de cobertura en salud (PROECOS) impulsado por el Ministerio de Protección Social (MPS) mediante la tarjeta de salud gratuita. El financiamiento del PROECOS y la tarjeta de salud provenía el $80 \%$ de los costos del presupuesto regular del MSP, el $20 \%$ restante contemplaba la movilización de recursos externos: Banco Mundial USD 97 millones por tres años, Unión Europea: USD 34 millones, Bélgica: USD 6 millones, España: USD 4 millones ${ }^{(10)}$. En el 2005 se creó la Secretaría de los Objetivos del Milenio (SODEM) ${ }^{(11)}$ para administrar el AUS, la misma que fue cerrada en el 2007 en el gobierno del Presidente Rafael Correa debido al cambio de visión en salud.

El Gobierno del Presidente Correa (2007-2016) propuso transformar el sistema de salud en un "sistema de acceso universal, gratuito y de excelente calidad", propuesta que puso en marcha desde el inicio de su período mediante la gratuidad progresiva y la declaración de emergencia para mejorar la infraestructura e incrementar el equipamiento, medicinas y recursos humanos en salud. En abril del 2008, el MSP presentó al ejecutivo la propuesta de transformación sectorial de salud en el Ecuador (TSSE) trabajada en conjunto con el Consejo Nacional de Salud (CONASA), Secretaria Nacional de Planificación (SENPLADES), IESS, FFAA y Policía Nacional, Ministerio Coordinador de Desarrollo Social (MCDS) y el apoyo técnico de la OPS/OMS, UNFPA y UNICEF ${ }^{(12)}$.

Los ejes de la TSSE en función del SNS son $7^{(13)}$.

1. Fortalecimiento de la autoridad sanitaria nacional, 2. Administración y Gestión del SNS, 3. Modelo de Atención integral y red de servicios públicos de salud, 4 Financiamiento del SNS. 5. Control y Monitoreo del SNS. 6. Sistemas de Gestión de Información en Salud 7. Participación ciudadana y control social.

Los ejes de la TSSE se vieron respaldados y fortalecidos con la aprobación de la nueva constitución, y en armonía con el modelo de gestión, la reforma democrática del Estado y las competencias del MSP. El modelo de gestión del MSP es de alta desconcentración y baja descentralización. Al nivel central le corresponde la formulación de la política pública de salud, regulación, planificación y control, ejerciendo de esta manera la rectoría sobre el SNS. Una de las propuestas centrales de la TSSE fue el desarrollo y consolidación de la "Red Pública Integral de Salud". La TSSE también definió el Régimen del Derecho a la Salud, que consiste en identificar las prestaciones garantizadas de salud en función de criterios de frecuencia, evitabilidad, capacidad resolutiva y consideraciones estratégicas ${ }^{(13)}$.

\section{Factores Políticos, Sociales y} Económicos

El escenario político del Ecuador en el período 2000-2007 [para el estudio se revisa desde el 2005], se caracterizó por una gran inestabilidad política (7 presidentes entre 1995-2005), con graves problemas de gobernabilidad, violencia social, golpes de estado y derrocamientos, inestabilidad administrativa y falta de continuidad de políticas. Salud no fue la excepción (9 ministros de salud entre el 2001 y el 2005), la inestabilidad afectó las potenciales reformas, evidenciando políticas públicas escasas o coyunturales y contradictorias ${ }^{(13)}$.

El liderazgo en salud también fue objeto de pugna de poderes, es así que en el período del 2003-2005, (Presidencia de Lucio Gutiérrez), el Vicepresidente Alfredo Palacios, lideraba la propuesta del AUS, la Primera Dama coordinaba el frente social con acciones en salud y el Ministro de Salud lanzó el Programa de Extensión de Cobertura en Salud (PROECOS).

La tendencia general de las gestiones ministeriales antes del 2007 fue la falta de presupuesto para los programas regulares e inversión en salud, generando préstamos a organismos internacionales para cumplir con los programas de inmunizaciones, atención materna infantil, entre otros.

En el 2007 (Presidencia de Rafael Correa) se inicia un proceso de priorización social, con la decisión política de posicionar la Salud como "derecho". La estabilidad política y gestión continua de los Ministros de salud ( 3 en el período 2007-2014) fue un factor decisivo para lograr avances en la universalidad y estructura del SNS. Santos, C., en su estudio The Politics of Health Reform in Ecuador $2016^{14}$, concluye que "Esta continuidad en la administración burocrática explica en gran medida la implementación exitosa de dos reformas legales importantes, programas de transferencias de efectivo, programas de becas y la construcción de varias instalaciones de salud estatales"

El alto nivel de aceptación y popularidad, el capital político y liderazgo del Presidente Correa sumado a la deslegitimación de los partidos políticos tradi- 
cionales y el apoyo ciudadano permitió que la Salud sea uno de los ejes centrales de las propuestas constitucionales en el marco de los derechos y que fuese aceptada y aprobada por la ciudadanía en el Referéndum del 2008.

A partir del 2013 con la caída del precio del petróleo se evidencian dificultades para el financiamiento de insumos, medicamentos y contratación de personal, el Gobierno Nacional a fin de no poner en riesgo la sostenibilidad de la gratuidad, incrementó nuevos impuestos para asignación a salud a productos de consumo, alcohol, cigarrillos, entre otros, los mismos que siguen siendo insuficientes para la creciente demanda de atención.

El Consejo Nacional de Salud (CONASA) y el MSP promovieron los Congresos por la salud y la vida (COSAVI), espacio democrático de veeduría y consulta nacional del sector salud y sociedad civil. En el II COSAVI en Guayaquil - 2004 se debatió la ley del SNS; en el III Congreso en Cuenca-2007 se trabajó la propuesta de Salud en la Nueva Constitución y el IV Congreso en Loja-2009 se presentó la TSSE y los avances de su implementación ${ }^{(15,16)}$.

Con la promulgación del código penal en el 2011, por el cual aparentemente se vería afectado el ejercicio de la práctica médica y la formulación del código de la salud, sin el suficiente consenso y participación de los asociaciones gremiales, sindicatos y veedurías sociales, se crea una brecha entre la autoridad sanitaria y los profesionales de la salud, realizándose manifestaciones y movilizaciones pacíficas de "resistencia", que sumado a otros grupos sociales y partidos de oposición, se genera un nuevo escenario político de inconformidad con las políticas del Gobierno, siendo necesario la intervención del Ejecutivo en el llamado al "diálogo".

\section{Rectoría}

En la Constitución del 2008, el MSP se consagra como rector del sector salud (3), pero el ejercicio de esa rectoría involucra el liderazgo y desarrollo de políticas, normativas, leyes que requieren ser legitimadas con la participación sectorial y social.

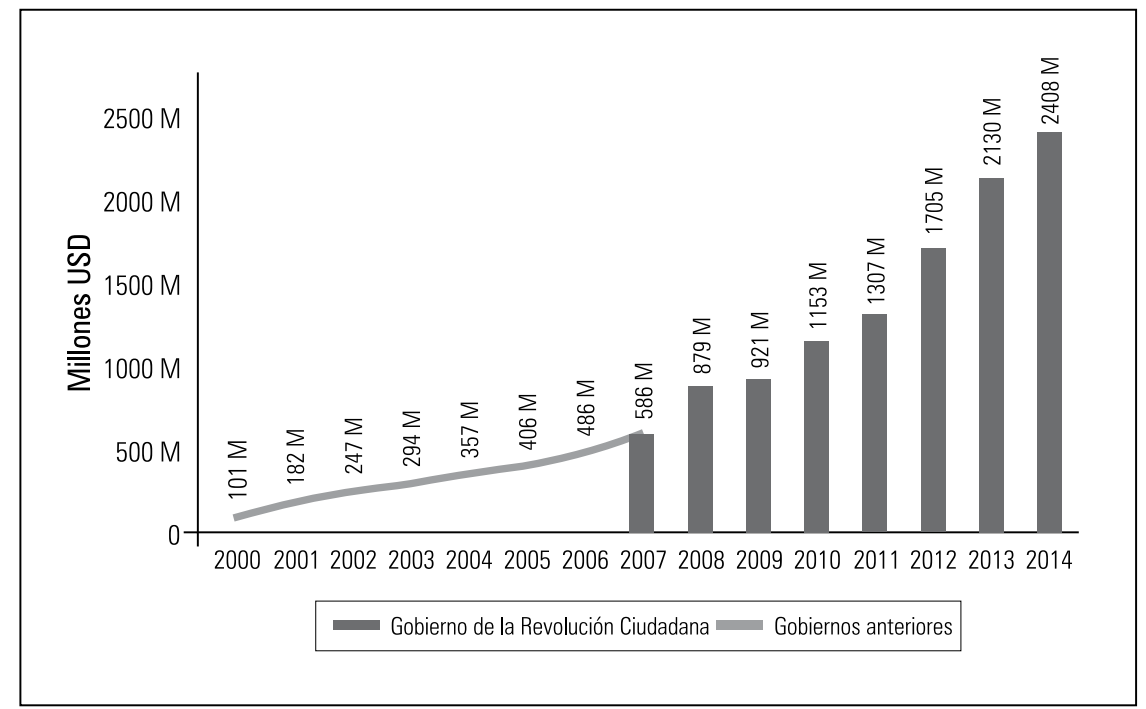

Figura 1. Presupuesto en USD ejecutado en salud. MSP.2000-2014. Fuente: eSIGEG, Ministerio Finanzas. Elaborado por Coordinación General de Planificación del MSP 2015.

Las mayoría de los entrevistados concuerdan que el MSP no ejercía la rectoría o era muy débil antes del 2007 y es a partir de ese año que se recupera o fortalece; consideraron que factores como la planificación de la TSSE en coordinación con el CONASA, la formulación de políticas y control epidemiológico en emergencias y pandemias y la paz laboral (periodo 2007-2010 no se registraron paros ni huelgas por primera vez en la historia del MSP), fueron estratégicos para el reconocimiento y conducción de la rectoría del MSP; sin embargo, también opinaron que esa rectoría no fue sostenida en el tiempo y sufre un revés a partir del 2010, percibiendo que la rectoría de salud la asume el Presidente de la República, que es quien empieza a tomar decisiones técnicas y políticas en salud. Así: "A partir del año 2007 realmente se recupera no solamente la institucionalidad del Ministerio de Salud Pública, sino además se construye la rectoría en el sistema nacional de salud...lamentablemente a partir de mediados del 2010, cae sustancialmente la rectoría del Ministerio de Salud en un proceso que duró alrededor de dos años... a partir del 2012 se intenta recuperar esa rectoría" (E5).

"La gobernanza se la ha abordado como hecho más burocrático y regulatorio que como el hecho de conseguir consensos y trabajar esos consensos... ese es quizás una de las debilidades que ocurren en el 2015 que no se ha podido avanzar o fortalecer la rectoría... Falta que haya libertad de decisión por parte de la autoridad sanitaria y líderes del sector salud. Quien ejerce es el presidente". (E3)

\section{Financiamiento}

El sistema de financiamiento de la salud en el Ecuador es mixto, el público (MSP) de fuente tributaria, la seguridad social (IESS,ISSFA, ISSPOL) financiado por contribuciones y el privado con seguros privados y gasto de bolsillo ${ }^{(1)}$.

El presupuesto del MSP mantuvo un incremento constante desde el 2001 (151,7 m) hasta el 2006 (561 m), pasando del 3,4\% del presupuesto general del estado al $6,6 \%$ y del $0,9 \%$ al $1,4 \%$ del PIB respectivamente. (OPS 2008) ${ }^{(9)}$. A partir del 2007 el presupuesto se incrementa considerablemente de $586 \mathrm{~m}$ en el 2007 a $921 \mathrm{~m}$ en el 2009, prácticamente al doble en comparación al 2005 y continúa creciendo a 1,4 m en el 2011 y $2.4 \mathrm{~m}$ en el $2014^{17}$. (Figura 1).

"Nosotros hemos ido de tener un presupuesto de 580 millones aproximadamente en el año 2007, a más de 2400 millones en el 2014 y un presupuesto si- 
milar en el 2015, son más de 11 mil millones de dólares que se han invertido en el sector salud, solamente un año de inversión en nuestro gobierno, en el 2014 equivale a la inversión que se ha realizado en salud, por parte de los cuatro gobiernos anteriores". (E11)

Según las cuentas nacionales, el gasto total en salud se situaba en el 2004 entre el $4 \%$ y el $5 \%$ del PIB proviniendo un $50,4 \%$ del gasto del sector público y un $49,2 \%$ del sector privado. En el 2010, el gasto total de salud corresponde al 8,6\% del PIB. El sistema público en su conjunto alcanza para 2011 una inversión de 216 USD per cápita en salud, correspondiendo a una participación en el PIB de 5\%, con elevación progresiva de los gastos del MSP y seguros sociales. En el año 2014, el gasto de consumo final de la salud con respecto al PIB alcanzó el 8,3\%, observándose un incremento acumulado de 2,8 puntos porcentuales entre el período 2007 y el $2014^{(17,18)}$.

En la figura 2 se muestra el porcentaje de participación del gasto público en salud respecto al PIB comparativamente entre el MSP y el IESS, mostrando el incremento sostenido en la inversión pública y el incremento proporcional al PIB, mientras que en la seguridad social se ha mantenido casi constante entre el 2008 y 2010.

Las opiniones de la mayoría de los entrevistados convergen en que el financiamiento a cargo del Estado a partir del 2007 obedece a un cambio de visión política y a una Constitución garantista del derecho a la salud, pero hay opiniones divergentes en las que se cuestiona no haber cumplido con la transitoria de la Constitución, de incrementar hasta el 4,5 $\%$ del PIB y no haber logrado cubrir necesidades de recursos humanos, medicamentos y atención de las enfermedades catastróficas: "El financiamiento ha sido importante, ha sido generoso, pero no ha cumplido el mandato constitucional, está por debajo del 4.5\%". (E7)

\section{Prestación de Servicios}

La propuesta del AUS y el programa PROECOS ${ }^{(8)}$ que contemplaba la atención gratuita a los quintiles 1 y 2 de pobreza mediante la tarjeta de salud, implicaba mejorar la capacidad de las unidades operativas para cubrir la demanda potencial de atenciones a los asegurados, para esto se desarrolló un proceso de licenciamiento de las unidades operativas: "...teníamos un plan de licenciamiento, habilitación y acreditación de salud con

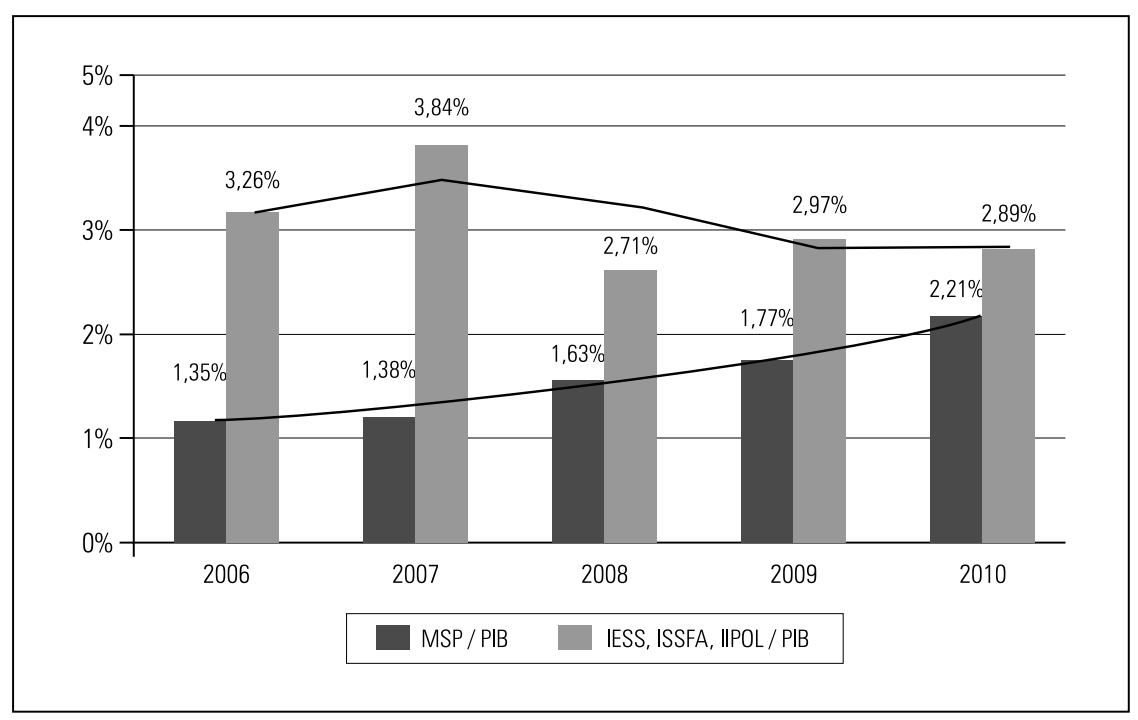

Figura 2. Porcentaje de participación del gasto público en salud respecto al PIB. Ecuador, 20062010. Fuente MSP 2010. cobertura no total, que esto es lo ideal como principio filosófico humano y también de populismo político, yo no creo que se deba dar cobertura universal sino una cobertura parcial, para primero, segundo, y parte del tercer quintil de la pobreza y el cuarto y el quinto que lo atienda a través de prepagas para poder dar cobertura y mantener un equilibrio entre lo público y lo privado". (E1)

El incremento de establecimientos de salud fue relevante en los últimos años. Desde el 2007 al 2010 se crearon 132 unidades de salud, 497 se remodelaron, 13 hospitales fueron ampliados, remodelados y equipados (MCDS 2011).

Las atenciones de salud en el MSP se incrementaron de manera considerable producto de la eliminación de barreras de acceso económico, geográfico y cultural, registrándose $14,372,251$ en el 2006, $19,779,874$ en el 2009 y 38,088,410 en el 2013.

La red pública integral de salud constituye el eje fundamental para la universalidad y consolidación del sistema nacional de salud, su articulación con la red complementaria ha permitido dar respuesta a la alta demanda aprovechando la capacidad instalada de la red privada con y sin fines de lucro, reduciendo inequidades, listas de esperas, sumado a los beneficios de la alianza público privada con mayor tecnología, especialistas, entre otros...

"Para el gobierno es una herramienta más que útil, le ha dado cobertura sin dinero, al instante que se produce la patología, eso le da una fuerza política a cualquier gobierno, porque las entidades privadas somos congruentes con la filosofía del gobierno, y yo diría que el $80-90 \%$ se hacen a un menor costo, con menor morbilidad, con menor tiempo de ocupación, con más eficiencia y con más humanismo". (E1)

Sin embargo, la excesiva y no planificada derivación de lo público a lo privado, ha puesto en riesgo la sostenibilidad del sistema al pagar altas sumas de dinero facturado por las entidades de la red pública y complementaria, y en los últimos años la falta de pago oportuno a estas instituciones ha limitado la atención a pacientes derivados del MSP. 
El Modelo de Atención Integral de Salud (MAIS) es un conjunto de políticas, estrategias, lineamientos y herramientas enfocadas en la salud familiar, comunitaria e intercultural que incorpora la estrategia de la APS (Atención Primaria en Salud) renovada para complementar, organizar y descongestionar el SNS (MAIS/ MSP) ${ }^{(13,19,20)}$.

Para la implementación del MAIS en el 2007 se fortaleció el I nivel de atención y se constituyeron 1792 Equipos Básicos de Atención de Salud (EBAS) integrados por médicos, enfermeras, odontólogos que durante el 2008-2009 visitaron 2’024,000 familias con acciones de promoción, prevención e identificación de factores de riesgo. En el 2010 se desintegraron los EBAS y el recurso humano pasó a cubrir la demanda y el abarrotamiento de los servicios hospitalarios. En el 2012 el MSP contrató a 1700 Técnicos de Atención Primaria de salud (TAPS) que se integrarían a los servicios de salud primarios.

La mayoría de los entrevistados concuerdan que la APS y el modelo de atención no está cumpliendo con sus objetivos y se ha dado excesiva prioridad a la atención hospitalaria y a la medicalización de la salud, debido principalmente a la injerencia política y directa del Ejecutivo sobre las decisiones de la institución rectora en salud.

"El modelo con base en APS se implementa en los primeros años pero se desvía hacia la hospitalización y medicalización de la salud... La demanda ahora es por calidad..." (E4)

"Se ha abandonado seriamente la atención primaria y digo con mucho dolor que el Presidente es quien ha propiciado que el hospital es el templo, ese concepto o esa confusión de que salud es medicina...en el MSP se habla menos de salud pública, menos de las funciones esenciales, se habla de acreditación hospitalaria, Mi hospital, Mi farmacia". (E3)

\section{Recursos humanos en salud}

En el 2007 para la implementación del MAIS con enfoque comunitario se contrataron más de 4500 profesionales, pasando la tasa de médicos de 14×10,000 habitantes en el 2006 a 16,9 por 10,000 habitantes en el 2009: "La tasa de médicos, enfermeras, obstetrices, y odontólogos por 10,000 habitante que existen en promedio de todo el país es de 32 por 10,000 habitantes, lo cual es muy bueno, está sobrepasando la mínima que la OPS dice que es 25 pero en área rural es muy preocupante encontrar que solo hay 8 por cada 10,000 habitantes" (E12)

Los siguientes años 2010-2014 se continuó con el incremento progresivo y redistribución estratégica del recurso humano, mediante iniciativas como el bono geográfico, el plan de retorno de los profesionales ecuatorianos en el exterior, convenios con Cuba para la inclusión de médicos familiares a la red de servicios de salud, entre otros. Pese a todo esto, los entrevistados consideran que la brecha de especialistas y la distribución inequitativa se mantuvo, y en el caso de las áreas de salud pública se han visto seriamente debilitadas al orientar las políticas de recursos humanos (Rhus) a la atención y no a la prevención.

"Se incrementan los Rhus pero distribuidos inequitativamente y con desvalorización y marginación del recurso con experiencia en salud pública...había en el MSP en el periodo inicial de la gestión gente con una trayectoria interesante $y$ el MSP en el 2011 sin ningún empacho y muy a la ligera se desprendió de personas valiosísimas, por ejemplo, un área donde la debilidad es más notoria, es la de control y vigilancia epidemiológica". (E3)

\section{Buenas prácticas}

Las representaciones sociales han evidenciado las buenas prácticas que han contribuido a la implementación de reformas y transformaciones en el SNS, se resumen las 6 de mayor convergencia:

\section{a. Politización de la salud}

Colocar a la salud pública como una prioridad en la agenda política, convirtiéndose en la bandera de lucha para el cambio social, la reducción de la pobreza y las inequidades, para el "buen vivir" ha sido una de las prácticas más destacadas por los informantes como referente del pro- ceso de transformación del sector salud a partir del 2007 en el Ecuador.

"Colocar la salud pública y los problemas de salud de las personas en la agenda política, y que el Presidente de la República fue el principal abanderado de la salud pública ecuatoriana ...posicionar a la salud en la mente de los ecuatorianos y hacer que el ecuatoriano, piense y discuta hable más sobre salud, eso es politización de la salud" (E2)

\section{b. Decisión política de invertir en salud}

La firme decisión política de "invertir en salud" para garantizar un derecho humano fundamental, de acuerdo a la percepción de los entrevistados ha constituido un hito en la historia de la salud pública, demostrando que la voluntad política hace la diferencia.

"Como aspecto positivo, aunque no se haya logrado el 4,5\% del presupuesto como manda la Constitución creo que se ha demostrado que cuando hay una fuerte decisión política, si se puede incrementar la inversión social...porque un pueblo sano así lo ha reconocido el gobierno, aporta al desarrollo de país" (E6)

\section{c. Constitución Política para garantizar} la continuidad

La opinión mayoritaria de los entrevistados coincide en que la Constitución del 2008 es una de las más avanzadas de la región, define claramente la estructura y financiamiento del sistema, garantiza el derecho a la salud y constituye la mejor herramienta o "best practice" a ser recomendada para la construcción, consolidación y sostenibilidad de un SNS.

"Creo que evidentemente el marco constitucional es una fortaleza para exigir a cualquier gobierno que viniere, que la salud tiene una dimensión enorme y que tiene que estar financiada de una forma adecuada y que tiene que desarrollarse en función de esas líneas constitucionales." (E7)

\section{d. Modelo de atención integral de salud MAIS}

El modelo del sistema de salud del Ecuador está basado en la APS, respeta e integra los valores y tendencias culturales 
con participación comunitaria. Se encuentra desarrollado y normatizado, convirtiéndose en otra buena práctica.

"Creo que una cuestión fundamental para construir una salud pública democrática y que logre impacto, es que se avance hacia un proceso diferente de la salud que dé importancia a la promoción de la salud, a la prevención, a las políticas públicas soberanas, sin interferen-

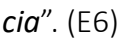

"Los EBAS permitieron un acercamiento de la salud a la comunidad, conocer su realidad e involucrarla para la búsqueda de soluciones en base a determinantes de la salud" (E10)

\section{e. Red pública integral de salud y complementaria}

La Red pública integral de salud como herramienta y eje fundamental del sistema permitiendo la extensión de la cobertura sin duplicación y en complementariedad con el sistema privado ha sido reconocida como una las buenas prácticas de gran impacto en la construcción de la universalidad.

"Un logro a destacar desde el primer momento, los primeros años de la revolución ciudadana, es el haber trabajado y eso que costó muchísimo esfuerzo, con otros sectores que incluso siempre han sido reactivos, y siempre fueron opositores al gobierno, por ejemplo con la Junta de Beneficencia de Guayaquil, con Sol$c a^{\prime \prime}$. (E3)

\section{f. Acercamiento a la comunidad.}

La estrategia de acercar a las autoridades de salud a la población en su ambiente de trabajo, vivienda, interactuar directamente con ellos escuchando, atendiendo y resolviendo los problemas localmente, ha sido de acuerdo a la percepción de los entrevistados una buena práctica para aterrizar las políticas públicas a las realidades de la población.

"Que los tres ministros de salud, tengan un contacto directo, cercano continuo con el Presidente, con el Congreso en general, con los municipios, con las personas, instituciones y personal de salud, ha hecho que el Ministerio de Salud sea más escuchado y más visto... y en las visitas constatar que se está cumpliendo lo que se les prometió...". (E3)

Lecciones aprendidas

Los entrevistados han identificado también las lecciones aprendidas, aquellas que no deberían repetirse y que han afectado la construcción o consolidación del sistema durante el periodo de estudio. A continuación, se describen las más frecuentes:

\section{a. Partidización de la salud.}

A diferencia de la politización considerada una buena práctica por colocar a la salud en la agenda política priorizada, los entrevistados identifican a la partidización como una lección aprendida por convertir a la salud en un "botín" político, en el cual los logros alcanzados no se reflejan en el beneficio a la población sino en rédito político que este genera.

"Yo creo que ese es quizás el pecado más grande que tenemos ahora, duele decirlo, pero para mí hemos pasado de trabajar por las necesidades de la gente a trabajar por la foto, por el video, por la promoción, por el mercadeo de una gestión". (E3)

"Yo creo que han sido decisiones politicas siempre con una visión proselitista, muchas de las acciones que se han realizado, han tenido primero la visión de retorno político" (E7)

\section{b. Injerencia política sobre decisiones técnicas.}

La injerencia directa sobre las decisiones competentes a la autoridad sanitaria que se vieron manifiestas a partir del 2010 , de acuerdo a la percepción de los informantes, es una práctica negativa que debilitó la rectoría del MSP, decisiones reactivas que en muchos casos solucionó problemas emergentes pero no atendió los problemas de fondo del sistema.

"El Presidente de la República debe ejercer a sus ministros, cuando el presidente cree saber más que el ministro del momento eso es un grave problema, él es un hombre inteligente, me parece que es el Presidente que necesitamos, pero él no sabe más que un buen técnico calificado, un salubrista, además el hecho que el escuche a su ministro, le da o le proporciona nuevas herramientas, otras perspectivas" (E3)

\section{c. Desvalorización del recurso humano}

Los entrevistados consideran que la desvaloración del recurso humano por la edad y tiempo de servicio sin considerar la experiencia y capacidades técnicas observado en los últimos años de gobierno, es una lección aprendida que no debería repetirse, ya que sumado a la carencia de especialistas ha incrementado la brecha de recursos humanos.

"Un nudo critico es el de recursos humanos no solamente por las brechas que había sino también por la difícil gobernabilidad para la política de recursos humanos en términos de formación de recursos humanos, que va a poner en riesgo toda la ganancia en algún momento en el Ecuador" (E12)

"La desvinculación en el 2011 de más de 400 profesionales de salud a nivel nacional sin criterio técnico y con medidas violentas mostró un desprecio al profesional de salud" (E10)

\section{d. Participación social para legitimar y sostener la propuesta}

La escasa o casi desaparición con el tiempo de los procesos participativos en el fortalecimiento del sistema y formulación de políticas y leyes, "institucionalizando o partidizando" la participación social, es otra lección aprendida.

"Si es que no se construye un proceso social desde la base no tiene resultado y lo que nos vayan a imponer como ley de seguridad social o como ley orgánica en sistema de salud no tendrá sentido cuando acabe este gobierno esto se irá abajo..." "...sin participación social no se podrá construir una salud diferente." (E12)

"Una de las grandes fortalezas que tenía el periodo previo y el inicio del periodo actual, ha sido la participación de todos los actores, el CONASA debió haberse fortalecido..." (E2)

Esta percepción coincide con lo que menciona Malo (21), es necesario: "ampliar y profundizar los mecanismos de participación ciudadana que permitan 
garantizar adecuados y eficientes niveles de control social en la gestión de la salud, pero, sobre todo, que generen condiciones para que los ciudadanos se involucren críticamente en la discusión de la política y consideren, de ser pertinente, reivindicar ante cualquier gobierno los beneficios de una reforma de esta naturaleza".

\section{e. Racionalizar y reorientar el gasto}

Una gran parte de los informantes relaciona que la elevada inversión en salud, calificada por ellos como "histórica y única", no se ve reflejada en el impacto sobre los indicadores de salud en especial de mortalidad materna, si bien es cierto la infraestructura de salud y equipamiento ha mejorado, es necesario racionalizar el gasto para hacer sostenible el sistema y reorientar la inversión más en la atención primaria, promoción y prevención.

"El hecho de pensar más en la economía de la salud al momento de la toma de decisiones no solo en términos puro económico, sino de eficiencia, en la incorporación de tecnología, las inversiones en otras áreas, que yo creo que han estado si no olvidadas, al menos relegadas, y me refiero a lo que tiene que ver con la promoción en salud, la epidemiologia, esa es la lección aprendida, que ha habido recursos pero no hemos utilizado los recursos para ese tipo de actividades para mayor rédito en bienestar de las personas."

\section{Desafíos para la consolidación del sistema de salud}

Las representaciones sociales consideran que las iniciativas de reformas han podido consolidarse a partir de la continuidad y estabilidad política desde el 2007, a pesar de ello, todavía el modelo de acceso universal con gratuidad no puede garantizar el ejercicio pleno del derecho a la salud universal, enfrentando aun muchos retos y desafíos, entre ellos:

- Poner a funcionar el sistema de salud que se consagra en la Constitución y darle sostenibilidad financiera a la universalidad y gratuidad.

- Decisión política de operativizar el MAIS por encima del modelo curativo.
- Desfragmentar al sistema mediante la operatividad eficaz y eficiente de la red pública y normatizar la alianza publico privada para utilizar eficiente y racionalmente la red complementaria. "La articulación con el IESS es uno de los principales desafíos que tiene la TSSE. La autonomía del IESS y el mantenimiento de las prestaciones de las contingencias de enfermedad y maternidad en la institución respaldada por la constitución, dificultan una mayor integración del SNS".(E10)

- Mejorar la calidad de la atención, con buen trato, calidez, pertinencia y oportunidad. "Ya vamos una década, ya nadie se acuerda de que había antes el cobro de la consulta...ahora quieren calidad,...los pacientes evalúan que se ha deteriorado la calidad de la atención, está menos personalizada y lógicamente eso también constituye otra barrera" (E4).

\section{CONCLUSIONES}

Como mencionan Loli (21) y Piña ${ }^{(23)}$, la Teoría de las Representaciones Sociales es un conjunto de conceptos, proposiciones y explicaciones originadas en el día a día, es por esto que en el presente estudio, la experiencia vivida, las principales reflexiones, posiciones y planteamientos de los actores que estuvieron involucrados en los procesos de reforma y TSSE en la última década, nos permitieron comprender cómo ha sido la evolución y los factores que influyeron en la decisión de pasar de un modelo de aseguramiento al de acceso universal de salud.

Dada la variedad de actores y periodos de participación política, las posiciones y opiniones han tenido convergencias $y$ divergencias al caracterizar cada componente del sistema, por ejemplo la rectoría, mientras que unos consideraron que a partir del 2007 se recuperó y fortaleció, otros planteaban que siempre hubo rectoría y otros simplemente opinaron que nunca se la ha ejercido, otro aspecto que mostró dicotomía ha sido el alcance de la universalidad en el acceso a servicios integrales y el financiamiento de los mismos, mientras que la percepción mayoritaria refiere un importante avance en la cobertura universal e inversión en salud, otros cuestionan la sostenibilidad financiera de la gratuidad y las deficiencias en el acceso a servicios integrales.

Algunas consideraciones finales en el marco de la percepción mayoritaria de los entrevistados sobre la evolución del sistema de salud del Ecuador durante el período 2005-2014 son:

- El sistema de salud del Ecuador se caracteriza por ser un sistema mixto, segmentado y fragmentado. Por mandato constitucional es de acceso universal y gratuito, la mayoría de los entrevistados concluye que desde el 2008 se encuentra en proceso de universalización, pero aún no se ha logrado ni consolidado el sistema.

- La mayoría de los actores coinciden en que la rectoría si bien es cierto esta asignada a la autoridad sanitaria, ésta debe fortalecer el liderazgo y lograr la legitimización por parte del sector y sociedad civil.

- La mayor convergencia es el reconocimiento de la alta y prioritaria inversión en salud que jamás se había dado y de que el financiamiento está garantizado por el Estado de acuerdo a la Constitución; sin embargo, algunos entrevistados consideran que el impulso de la inversión en salud estaba vinculado al precio alto del petróleo y el compromiso político de fortalecer el aparato estatal, y que es necesario la racionalización del gasto para asegurar la sostenibilidad de la gratuidad.

- La mayoría de los entrevistados convergen en que la prestación de servicios debe orientarse al modelo de atención, retomar la APS, e implementar una política de recursos humanos para fortalecer la oferta pública.

- La percepción mayoritaria coincide en que los factores políticos, sociales y económicos que han influido a partir del 2007 han brindado un escenario favorable para las reformas e implementación de la TSSE, formular leyes e impulsar el código de salud, pero ésta no cuenta con la sostenida legitimidad ciudadana, por lo que la alteración o revés de estos factores pondría en riesgo los avances y consolidación del sistema de salud. 
- Posicionar a la salud como "derecho" en la Constitución de la República y colocarla como prioridad de Estado y de Gobierno ha sido una de las mejores "buenas prácticas" de acuerdo a los entrevistados, demostrando que "Invertir en salud es la mejor estrategia para alcanzar el desarrollo de un país".

- Una "lección aprendida" producto del consenso de las opiniones de los entrevistados es sin duda que la participación social en la construcción y veeduría del proceso, legitima, valida y sostiene los procesos de cambio y que sin la misma no se garantiza la continuidad y sostenibilidad de dichos cambios.

- Los principales desafíos del sistema de salud del Ecuador son la sostenibilidad financiera de la universalidad y gratuidad en consonancia con la calidad de la atención.

\section{REFERENCIAS BIBLIOGRÁFICAS}

1. Giovanella L, Ruiz G, et al. Sistemas de salud en América del Sur. En: Giovanella L, Feo O, et al. Sistema de Salud en Suramérica: desafíos para la universalidad la integralidad y la equidad. Rio de Janeiro: Instituto Suramericano de Gobierno en Salud. 2012; p. 22-65

2. Christopher D, et al. Investigaciones para una cobertura sanitaria universal. Informe sobre la salud en el mundo 2013. Ginebra: Organización mundial de la salud; 2013. p. 6-10

3. Constitución Política de la República del Ecuador. Título II Derechos; Título VII Régimen del buen vivir, sección segunda Salud. Ecuador: Constitución política de la República del Ecuador; 2008.

4. Organización Panamericana de la Salud, Ministerio de Salud Pública. La salud en la nueva constitución. Ecuador: OPS/OMS, MSP; 2008.

5. González T, Cano A. Introducción al análisis de datos en investigación cualitativa: Concepto y características (I) Nure investigación, No. 44; Enero-Febrero 2010.

6. González T, Cano A. Introducción al análisis de datos en investigación cualitativa: Tipos de análisis y proceso de codificación (II) Nure investigación, No. 45; Marzo-Abril 2010.

7. Mora M. Citando a Dario Páez, en la Teoría de las representaciones sociales de Serge Moscovici. Atenhea digital-num 2; 2002.

8. Moscovici S. El Psicoanálisis, su imagen y su público. Buenos Aires: Ed. Huemul. 2da. Edición; 1979.

9. Organización Panamericana de la Salud. Perfil de Sistema de Salud: Ecuador, monitoreo y análisis de los procesos de cambio y reforma". Washington, D.C: OPS, Tercera Edición; 2008

10. Hexagon Cónsultores Informe Proyecto PROECOS (entregado a MEF para ingreso al SNIP). Ecuador: MSP; 2005

11. Lucio R, Villacrés N, Henríquez R . Sistema de salud de Ecuador. Salud Pública México. México: 2011

12. Carranza C, Cisneros M. Hacia un sistema de protección social más inclusivo en el Ecuador. Chile: CEPAL, Serie Políticas Sociales; 2014

13. Ministerio de Salud Pública. Transformación del Sector Salud. Ecuador: MSP; 2009

14. Santos, C. The Politics of Health Reform in Ecuador. Health Care Policy and Reform; 2016

15. Organización Panamericana de la Salud Ecuador, III Congreso por la salud y la vida, Cuenca 2008. Ecuador: 2008. Disponible en: http:// www. paho.org/ecu/index.php?option=com content\&view=article\&id=79:iii-congreso-saludvida\&Itemid $=972$

16. Organización Panamericana de la Salud Ecuador, 4to Congreso por la salud y la vida Edmundo Granda, Loja 2009. Ecuador: 2009. Disponible en: http://www.paho.org/ecu/index.php?option=com content\&view=article\&id=154:4to-congreso-saludvida\&Itemid $=290$

17. Viteri Díaz, G. Situación de la salud en el Ecuador en Observatorio de la Economia Latinoamericana, Número 77, 2007. Disponible en: http://www.eumed.net/cursecon/ecolat/index.htm 2007.

18. Instituto Nacional de Estadistica y Censos. [internet]. Ecuador: Cuentas satélites de los servicios de salud 2007-2014. [actualizado diciembre 2017] Disponible en:http://www.ecuadorencifras.gob.ec/ cuentas-satelite-de-los-servicios-de-salud/

19. Ministerio de Salud Pública. Manual del Modelo de atención integral de salud familiar, comunitario e intercultural MAIS-FCI. Norma Modelo de Atención Integral en Salud SEPSS - MSP. Ecuador: MSP; 2010

20. Ministerio de Salud Pública Modelo de Atención Integral en Salud Familiar, Comunitaria e Intercultural Ministerio de Salud Pública, Subsecretaria de Extensión de la Protección Social en Salud. Documento original. Ecuador: MSP;2008

21. Malo-Serrano M, Malo-Corral N. Reforma de Salud en Ecuador: nunca más el derecho a la salud como un privilegio. Rev Perú Med Exp Salud Publica. 2014;31(4):754-61

22. Loli A. Representaciones sociales de la enseñanzaaprendizaje de la investigación en enfermería en la facultad de medicina. UNMSM 2012. Perú: UNMS; 2014.

23. Piña Osorio J, Cuevas Cajiga Y. La teoria de las representaciones sociales: su uso en la investigación educativa en México. Perfiles Educativos 2004; 26(105-106): 102-124. 